\title{
La huerta escolar como estrategia en el desarrollo de competencias y el pensamiento científico.
}

\author{
Dilandia Nelly Tobar ${ }^{2}$ \\ Diana Johana Carabalí-Banguero ${ }^{3}$ \\ Deyanira Stella Bonilla ${ }^{4}$
}

Recibido: 08-05-2019

Aceptado: 17-08-2019

\section{Resumen}

La huerta escolar es una estrategia pedagógica que permite fortalecer la soberanía alimentaria, el cuidado del medio ambiente mediante prácticas agronómicas ecoamigables y la apropiación de competencias propias de las ciencias biológicas en el entorno, con el fin de que los estudiantes de la Institución Educativa Agroindustrial Valentín Carabalí, localizada en la Balsa, Buenos Aires (Cauca), adquieran competencias básicas y laborales por medio de experiencias interdisciplinarias bajo un modelo cooperativo-

\begin{abstract}
1. Artículo de investigación científica y tecnológica. Este producto se adscribe dentro de la propuesta pedagógica "Proyecto Innovadores y transformadores de cambio ambiental a través de la reforestación de la cuenca hidrográfica Agua Caliente, la huerta escolar y el reciclaje" que adelantan docentes de la Institución Educativa Agroindustrial Valentín Carabalí, Sede Principal, ubicada en el corregimiento La Balsa, Municipio Buenos Aires (Cauca, Colombia), que es apoyado por la institución educativa.
\end{abstract}

2. Docente de Aula, Institución Educativa Agroindustrial Valentín Carabalí Sede Principal, Cauca, Colombia. Licenciada en Biología y Química (Universidad Santiago de Cali).

ORCID: https://orcid.org/0000-0001-8135-2283

Correo electrónico: ntobar27@hotmail.com

3. Docente de Aula, Institución Educativa Agroindustrial Valentín Carabalí Sede Principal, Cauca, Colombia. Magister en Ciencias-Biología (Universidad del Valle). Bióloga (Universidad del Valle).

ORCID: https://orcid.org/0000-0001-5383-4357

Correo electrónico: dianajohana16@hotmail.com

4. Docente de Aula, Institución Educativa Agroindustrial Valentín Carabalí Sede Principal, Cauca, Colombia. Especialista en Gerencia Informática (Corporación Universitaria Remington), Especialista en Informática y Multimedios (Fundación Universitaria Los Libertadores), Licenciada en Ciencias Agropecuarias (Universidad del Valle).

ORCID: https://orcid.org/0000-0003-2212-1279

Correo electrónico: deyastellabonilla@gmail.com 
colaborativo, y así fortalezcan los contenidos aprendidos dentro del aula mediante prácticas vivenciales. Para ello, se llevaron a cabo las fases: preparación del terreno y diseño de figuras geométricas, recolección de datos en la huerta escolar, creación de guías de trabajo y análisis de su efecto en el aprendizaje significativo de los estudiantes y los docentes.

Palabras clave: competencias para la vida, geometría, hortalizas, método científico, reciclaje, sensibilización ambiental.

\section{The school garden as a strategy for the development of skills and scientific thinking}

\section{Abstract}

The school garden is a pedagogical strategy to strengthen food sovereignty, care for the environment through eco-friendly agronomic practices and use biological sciences skills specific to the surroundings. Using this strategy, the students of the Agroindustrial Educational Institution Valentín Carabalí, located in La Balsa, Buenos Aires (Cauca), acquire basic and labor skills through interdisciplinary experiences under a cooperativecollaborative model and thus strengthen content learned within the classroom through experiential practices. This strategy was carried out in the following phases: preparation of the land and design of geometric figures, data collection in the school garden, creation of work guides and analysis of the effect on the significant learning of students and teachers.

Keywords: life skills, geometry, vegetables, scientific method, recycling, environmental awareness. 


\section{Introducción}

La huerta escolar es un sistema ecológico que permite educar a los estudiantes en la comprensión de las interacciones y dinámicas ecológicas que ocurren en estos agroecosistemas, a través de la indagación y realización de experimentos, con miras a desarrollar una capacidad analítica que les permita extrapolar estos conocimientos en otros ámbitos de las ciencias naturales. De este modo, se motiva al estudiante a que realice una reutilización de materiales, aprovechamiento y cuidado del entorno (Botella-Nicolás, Hurtado-Soler, \& Cantó-Doménech, 2017). Se resalta la importancia de la huerta escolar en la educación en valores que conlleven a fortalecer la identidad, mostrando apropiación y respeto por el entorno (García-Ferrandis, Vilches-Peña, \& García-Ferrandis, 2017).

Se hace uso de la interdisciplinariedad planteada en el proyecto educativo institucional, apoyado en el artículo 67 de la Constitución Política y en el artículo 5, en sus numerales 9 y 13, de la Ley 115 de 1994. Se busca desarrollar competencias básicas y laborales mediante un aprendizaje significativo que enlace la protección del medio ambiente con prácticas agrícolas, que fortalezcan el conocimiento impartido en las aulas.

En el desarrollo de competencias, se requiere la comprensión de conceptos y teorías científicas, a través de métodos que fomenten un pensamiento analítico y crítico al analizar la validez de la información, contrastando las hipótesis con los datos obtenidos, teniendo presente el compromiso con la comunidad, ya que se debe fomentar una cultura científica que beneficie la sociedad (Ministerio de Educación Nacional [MEN], 2003).

La huerta escolar contribuye al mejoramiento de la calidad de vida de los estudiantes y sus familias, al generar conocimientos agrícolas que pueden utilizar en su vinculación al mercado laboral, planteando alternativas de negocios desde su entorno y garantizando la soberanía alimentaria y el cuidado del medio ambiente por el empoderamiento de su entorno. 
En el desarrollo de las actividades en la huerta escolar, se fomenta un ambiente de trabajo interdisciplinario, en el que los estudiantes son autónomos en su proceso de enseñanza-aprendizaje (Botella et al., 2017). De este modo, se pretende aplicar los conceptos aprendidos en el aula de clases en la creación y manejo de una huerta escolar, buscando generar una actitud positiva y el desarrollo de pensamiento crítico a través de las labores agrícolas, la interacción con la comunidad y el uso de las tecnologías de la información (TIC).

\section{Metodología}

Guiado por el modelo de constructivismo social etnoeducativo, se realizaron actividades que implicaban trabajo cooperativo-colaborativo. Los estudiantes aplicaron los conocimientos impartidos por los docentes en las áreas de desarrollo comunitario, orientación vocacional, emprendimiento, ciencias naturales, química, matemáticas y tecnología e informática. Se implementó una huerta escolar en la Institución Educativa (IE) Agroindustrial Valentín Carabalí, ubicada en el corregimiento de la Balsa, municipio de Buenos Aires, Cauca. La población estuvo representada por 44 estudiantes del grado $8 .^{\circ}$ y seis docentes.

En un área de $20 \mathrm{~m} 2$, se construyeron eras con figuras geométricas (triángulos, rectángulos, circunferencia) y diseños artísticos (mariposa). En la delimitación de las eras se emplearon botellas de polietileno teraftalato (PET, por sus siglas en inglés) de $400 \mathrm{~mL}$, obtenidas a través del reciclaje en la tienda escolar. Se sembró cilantro (Coriandrum sativum L.), repollo (Brassica oleracea var. Capitata L.), remolacha (Beta vulgaris L.), cimarrón (Eryngium foetidum), acelga (Beta vulgaris var. Cicla (L.) K. Koch) y estropajo (Luffa aegyptiaca Mill.) (figura 1). 


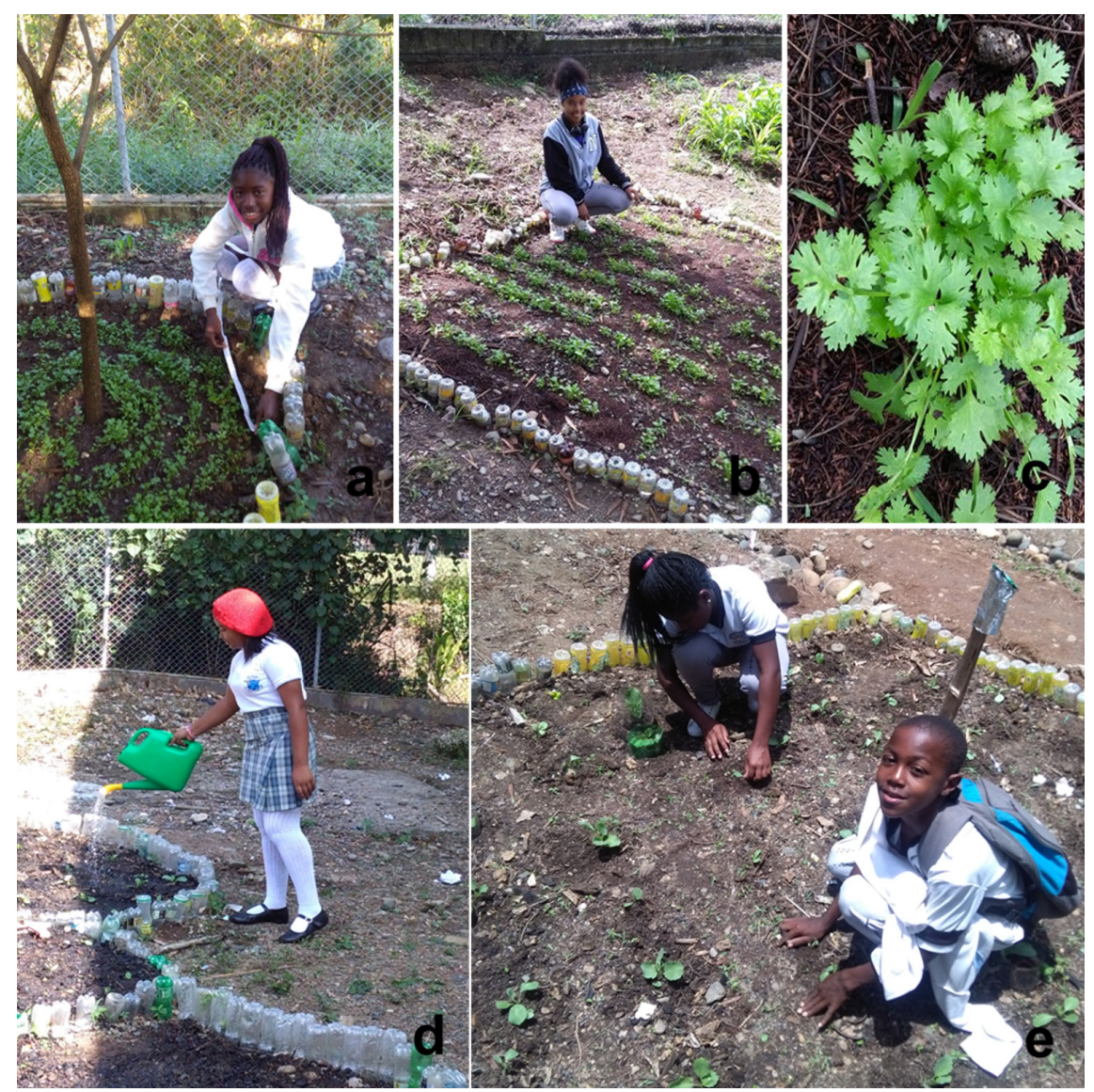

Figura 1. Establecimiento de la huerta escolar en la IE Agroindustrial Valentín Carabalí. a, b y c. Siembra de cilantro en una circunferencia y en un cuadrado; d. Siembra de remolacha en era con forma de mariposa; e. Siembra de repollo en era de forma cuadrada. Fuente: elaboración propia.

Los estudiantes emplearon técnicas agrícolas y ancestrales para el manejo de la huerta, como las siguientes: siembra de las hortalizas de acuerdo con las especificaciones de la variedad; riego cada 48 horas; control de plagas con insecticida organofosforado, realizando el menor número de aplicaciones posible; control manual de plantas arvenses; fertilización con úrea, roca fosfórica y tierra de carbonera. Las actividades se enfocaron en el aprendizaje por indagación para fortalecer la adquisición de competencias laborales. 
Para fomentar aprendizajes significativos en la huerta escolar, se realizaron las siguientes actividades experimentales:

1. Seguimiento semanal de la huerta en el que, en un libro de campo, se registraron variables como la altura de la planta, la forma de las hojas, el diámetro de la hortaliza, el porcentaje de germinación por metro cuadrado, el cálculo de la densidad por área, el crecimiento de las plantas, los dibujos y esquemas de los cambios de las plantas, el reconocimiento de principales plagas y enfermedades que afectan el cultivo (figura 2).

2. Mediante trabajo cooperativo-colaborativo, los estudiantes aplicaron las fases del método científico en la observación, planteamiento de hipótesis, experimentación y obtención de conclusiones de lo observado.

3. Resolución de guías de trabajo, que integran conocimiento de las distintas asignaturas. También presentaron un informe final por asignatura.

La didáctica de aprendizaje llevada a cabo en el aula de clases involucró actividades pedagógicas como presentación de recursos audiovisuales, elaboración de mapas conceptuales, elaboración de narraciones sobre la huerta escolar, prácticas de laboratorio, hojas de datos, uso de las TIC en la investigación de aspectos de las hortalizas sembradas y manejo de la huerta, enseñanza vivencial de valores ambientales y respeto por la biodiversidad. 


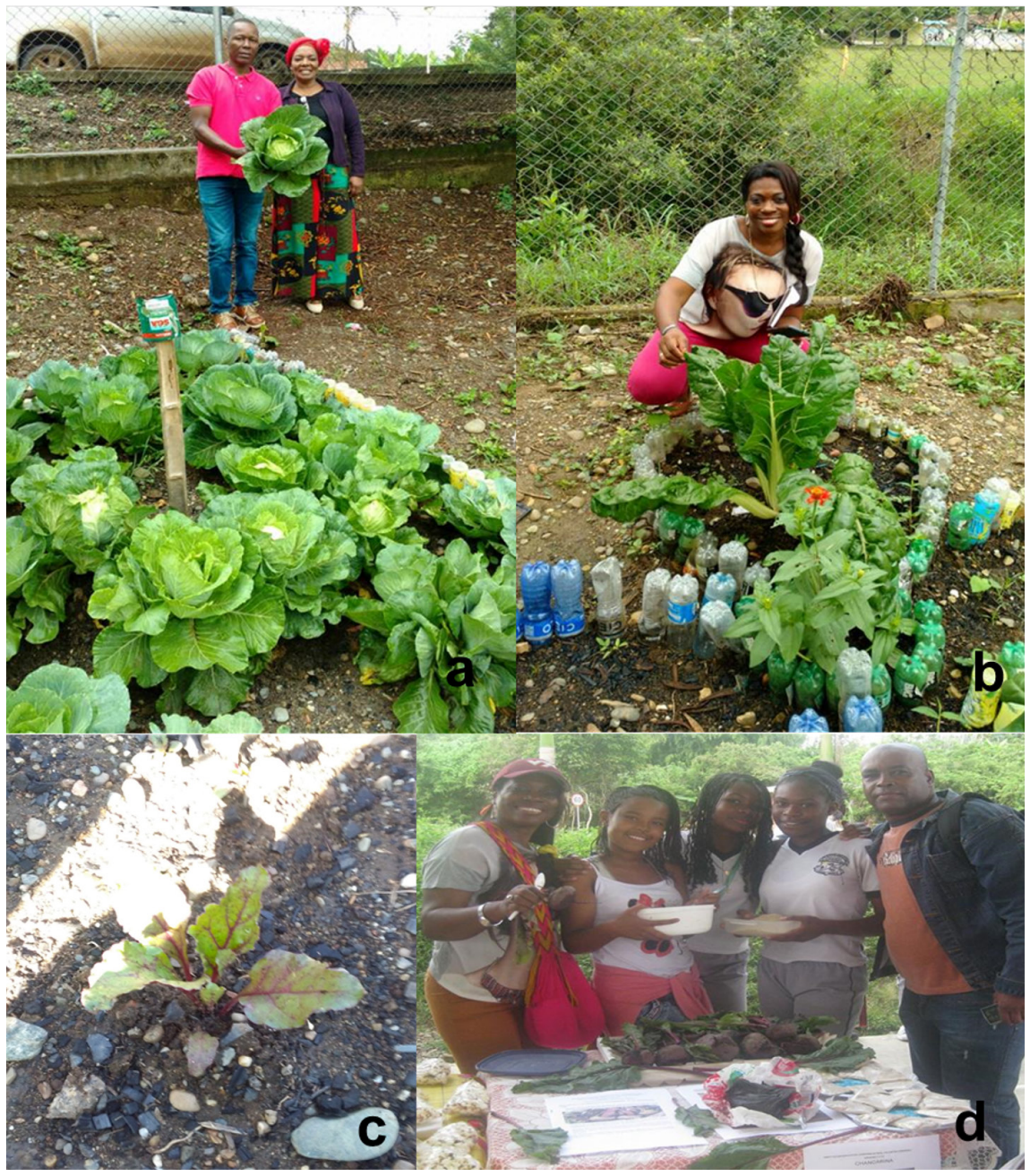

Figura 2. Huerta en etapa productiva. a. Repollo; b. Acelga; c. Remolacha; d. Comercialización de remolacha. Fuente: elaboración propia.

\section{Resultados}

Los estudiantes desarrollaron competencias laborales en la implementación de cultivos de hortalizas, destrezas en el manejo de instrumentos de medición, capacidad de análisis e interpretación de lo observado, y crearon registros como diarios de campo, na- 
rraciones, gráficos, tablas, dibujos, registros fotográficos y videos. El trabajo en equipo fortaleció la convivencia, tolerancia y aceptación de ideas contrarias a las propias. El proceso de aprendizaje se fortaleció por la interacción entre compañeros de clase; además, el desarrollo de las fases del método científico los motivó a realizar un trabajo riguroso y responsable.

Los recursos didácticos y materiales utilizados por los docentes facilitaron el proceso de aprendizaje del estudiante a través de la ejemplificación, retroalimentación de los saberes previos del estudiante, las visitas guiadas a la huerta para la recopilación de la información acerca de los cultivos y la realización de las prácticas planteadas. En la evaluación de los alumnos se realizó autoevaluación, heteroevaluación y coevaluación. Se tuvo en cuenta la teoría de las inteligencias múltiples, considerando el trabajo en equipo e individual, la participación, la disposición para las actividades agrícolas, la realización de actividades planteadas dentro del aula de clases y la socialización de los resultados obtenidos ante la comunidad educativa.

Los estudiantes autoevaluaron las actividades realizadas en la huerta escolar, al igual que dentro del aula de clases, lo que aumentó el interés por las asignaturas involucradas; estos se consideraron gestores de su proceso de aprendizaje. Además, reforzaron los conocimientos teóricos adquiridos en las aulas de clase mediante el trabajo en contexto reconociendo su entorno. Asimismo, socializaron a sus compañeros de clase las apreciaciones de cada uno y los resultados obtenidos en el aprendizaje cooperativo-colaborativo; también capacitaron a otros miembros de la comunidad sobre los beneficios de la siembra de hortalizas desde el punto de vista nutricional y su importancia para la soberanía alimentaria.

\section{Discusión}

La ciencia y la tecnología tienen una incidencia fundamental en el desarrollo de los pueblos y la vida cotidiana de las personas, 
permitiendo reconocer la complejidad de un mundo cambiante y desafiante. La ciencia brinda a las personas los conocimientos y herramientas necesarias para lograr comprender su entorno, y formar individuos capaces de innovar, observar y analizar todo lo que acontece.

El conocimiento científico trata acerca del desarrollo colectivo, en el que se da prelación a los trabajos con investigación, caminos recorridos para dialogar con otros y sacar conclusiones del trabajo. En la actualidad, al hablar de la ciencia, en singular, se habla más bien de disciplinas científicas, consideradas como cuerpos del conocimiento científico; hoy en día, las instituciones educativas deben saber que, en su seno, cohabita una serie de conocimientos que no solo vienen del plano académico y científico, sino que dentro de las comunidades también están inmersos los conocimientos preliminares con sus saberes ancestrales, propios de las culturas étnicas y populares. El educador debe formar a un estudiante que sea capaz de formular preguntas y soluciones con base en los saberes preconceptuales recogidos de cada estudiante.

Por esta razón, en las clases de ciencias naturales es importante brindar espacios que proporcionen en los estudiantes oportunidades de construcción de conocimiento, que será refinado y reafirmado en cada individuo al reconocer los diferentes puntos de vista, lo que se asocia con un aprendizaje significativo y crítico (MEN, 2003).

La interacción de los alumnos cuando trabajan de manera colaborativa permite obtener un mayor rendimiento en el aprendizaje. No obstante, la calidad del aprendizaje depende de factores como la heterogeneidad; el tamaño y la composición del grupo; la actividad asignada; el tema o conocimiento que deben aprender, y la participación del docente, que debe planear por anticipado estrategias para potencializar la productividad (Onrubia, Colomina, \& Engel, 2008). El trabajo colaborativo se fortalece con la participación activa de estudiantes con puntos de vista distintos que toman decisiones conjuntas y guías de trabajo. El aprendizaje cooperativo es otra forma de trabajo en grupo, en la que no hay una resolu- 
ción conjunta de la problemática para alcanzar una meta común, sino que se tienen metas individuales que se realizan de manera coordinada de acuerdo con la dirección del docente (Onrubia et al., 2008).

Durante la investigación se realizó un trabajo cooperativo-colaborativo, que implicó la asignación de tareas de manera coordinada, manteniendo la disposición de una resolución conjunta de las actividades planteadas en la investigación. El trabajo cooperativo hace parte de la didáctica, permitiendo integrar contenidos y métodos para la aplicación de los conocimientos adquiridos en contexto o en espacios virtuales, así como la reflexión e intercambio de saberes (Pereira-Almaguer, Díaz-Castillo, \& Pérez-Leyva, 2016).

La didáctica permite abordar los conocimientos científicos bajo un enfoque multidisciplinario, con la finalidad de mejorar el proceso de enseñanza-aprendizaje de las ciencias (Pereira-Almaguer et al., 2016). La interdisciplinariedad implica la creación de materiales didácticos integrados, que articulen conceptos de las distintas áreas para su aplicación en las aulas (Pereira-Almaguer et al., 2016). Con la transversalización del currículo, se logra incrementar la calidad del proceso de enseñanza-aprendizaje; los estudiantes adquieren conocimientos integrados que les facilitan la interrelación de conceptos, utilizando para ello la experimentación y las tecnologías de la información (Pereira-Almaguer et al., 2016).

La investigación, como estrategia para generar aprendizajes significativos, permitió evaluar la calidad del proceso educativo, al involucrar estrategias catalogadas como exitosas: la resolución de problemas, la retroalimentación y la autoevaluación formativa (Vázquez-Alonso \& Manassero-Mas, 2017). También se involucraron estrategias con grandes efectos en los estudiantes, como el aprendizaje cooperativo en pequeños grupos, en los que los estudiantes interactúan a través de sus puntos de vista y con la aceptación de ideas contrarias (Vázquez-Alonso \& Manassero-Mas, 2017).

Las aplicaciones de las estrategias planteadas están fundamentadas en el constructivismo social: los estudiantes aprendieron en 
sociedad, intercambiando ideas con sus compañeros, para llegar a un consenso a través de las actividades propuestas (Vázquez-Alonso \& Manassero-Mas 2017). Por otra parte, la ejecución de actividades en contexto permitió el desarrollo de las competencias que fueron evaluadas con distintos criterios. Se resaltó la importancia de la huerta escolar al propiciar un acercamiento a la naturaleza, así como por medio de la concientización sobre la conservación de los recursos naturales renovables y no renovables, la importancia del reciclaje y la preservación de la biota mediante un uso racional de agroquímicos.

\section{Referencias bibliográficas}

Aparicio, O.Y. (2018). La investigación escolar. Revista Interamericana de Investigación, Educación y Pedagogía, RIIEP, 11(2). https://orcid. org/0000-0003-3535-6288

Pereira-Almaguer, Y., Díaz-Castillo, R., \& Pérez-Leyva, G. (2016). El enfoque profesional interdisciplinar en el proceso de enseñanza-aprendizaje de la carrera Licenciatura en Educación, especialidad Biología-Química. Didasc@lia: Didáctica y Educación, 7(2), 37-56.

Botella-Nicolás, A. M., Hurtado-Soler, A., \& Cantó-Doménech, J. (2017). El huerto escolar como herramienta innovadora que contribuye al desarrollo competencial del estudiante universitario. Una propuesta educativa multidisciplinar. Vivat Academia, 139, 19-31. doi: http://doi. org/c89w

García-Ferrandis, I., Vilches-Peña, A., \& García-Ferrandis, X. (2017). Estrategias didácticas innovadoras en la enseñanza de las ciencias. Estudio de caso: los maestros del Patronato de Educación Rural de Valencia (1958-1985). Enseñanza de las Ciencias, 35(2), 109-126. 
Recuperado de https://www.raco.cat/index.php/Ensenanza/article/ view/324225/414842

Herrera, J.D. (2016). La relación escuela - comunidad: un análisis desde la teoría de sistemas a nueve experiencias de América Latina. Revista Interamericana de Investigación, Educación y Pedagogía, RIIEP, 9(1). DOI: https://doi.org/10.15332/25005421

Ministerio de Educación Nacional (MEN). (2003). Estándares Básicos de Competencias en Ciencias Sociales y Ciencias Naturales. Bogotá: MEN.

Onrubia, J., Colomina, R., \& Engel, A. (2008). Entornos Virtuales de aprendizaje basados en el trabajo en grupo y el aprendizaje colaborativo. En C. Coll \& C. Monereo (Eds.). Psicología de la educación virtual: aprender y enseñar con las tecnologías de la información y la comunicación (pp. 213-231). Madrid: Ediciones Morata.

Pérez, T.H.P. (2014) Colombia: de la educación en emergencia hacia una educación para el posconflicto y la paz. Revista Interamericana de Investigación, Educación y Pedagogía, RIIEP, 7(2). DOI: https://doi. org/10.15332/25005421

Tahull, J. (2016). Modernidad, educación y género. El proyecto inacabado. Revista Interamericana de Investigación, Educación y Pedagogía, RIIEP, 9(2), 159-178. DOI: https://doi.org/10.22490/25391887.1947

Vázquez-Alonso, Á., \& Manassero-Mas, M. A. (2017). Interdisciplinariedad y conceptos nómadas en didáctica de la ciencia: consecuencias para la investigación. Revista Eureka sobre Enseñanza y Divulgación de las Ciencias, 14(1), 24-37. Recuperado de https://revistas.uca.es/index. php/eureka/article/view/3003 